\title{
Focusing Attention on Decoding for Children With Poor Reading Skills: Design and Preliminary Tests of the Word Building Intervention
}

\author{
Bruce McCandliss \\ Weill Medical College of Cornell University \\ Isabel L. Beck, Rebecca Sandak, and Charles Perfetti \\ University of Pittsburgh
}

\begin{abstract}
This study examined the reading skills of children who have deficient decoding skills in the years following the first grade and traced their progress across 20 sessions of a decoding skills intervention called Word Building. Initially, the children demonstrated deficits in decoding, reading comprehension, and phonemic awareness skills. Further examination of decoding attempts revealed a pattern of accurate decoding of the first grapheme in a word, followed by relatively worse performance on subsequent vowels and consonants, suggesting that these children were not engaging in full alphabetic decoding. The intervention directed attention to each grapheme position within a word through a procedure of progressive minimal pairing of words that differed by one grapheme. Relative to children randomly assigned to a control group, children assigned to the intervention condition demonstrated significantly greater improvements in decoding attempts at all grapheme positions and also demonstrated significantly greater improvements in standardized measures of decoding, reading comprehension, and phonological awareness. Results are discussed in terms of the consequences of not fully engaging in alphabetic decoding during early reading experience, and the self-teaching role of alphabetic decoding for improving word identification, reading comprehension, and phonological awareness skills.
\end{abstract}

Requests for reprints should be sent to Bruce McCandliss, Sackler Institute, Box 140, 1300 York Avenue, New York, NY 10021. E-mail: bdm2001@med.cornell.edu 
The value of direct instruction in phonics ${ }^{1}$ has been supported in reviews of comparative classroom studies (Adams, 1990; Chall, 1987; National Reading Panel, 2000; Snow, Burns, \& Griffin, 1998). The value of such approaches and their emphasis on alphabetic decoding of letters to sounds is particularly evident in intervention studies with children identified as poor readers or at risk for reading failure (e.g., Foorman, Fletcher, Francis, Schatschneider, \& Mehta, 1998; Lovett, Ransby, Hardwick, \& Johns, 1989; Torgesen, in press). Characteristically, the most dramatic benefits of explicit phonics approaches have been demonstrated in measures of word and pseudoword reading, skills that form the foundation of the reading process (Gough, 1991; Stanovich, 1991; Torgesen et al., 1999).

Nevertheless, questions continue to be raised about phonics instructions and intervention at several levels: What are the characteristics of at-risk children who might benefit most from explicit phonics? Can intervention programs be adapted to target well-specified cognitive deficits in decoding skill? How is training in phonemic awareness best incorporated to enhance gains in decoding? Can reading comprehension benefit from an explicit phonics approach? Such questions require extensive measurements of reading subskills, within fine-grained analyses of decoding abilities and across broad arrays of reading skill measures, and careful consideration of the instructional activities that are embedded in decoding programs. Another important question-How much explicit phonics is required for children with poor decoding skills? - requires studies that carefully examine the impact of particular amounts of explicitly described instructional activities on developing reading skills. This study addresses such issues in the service of assessing one program for improving decoding skills.

One goal of our study was to develop a cognitive profile of children who had been in regular reading classrooms but had failed to acquire adequate decoding skills. We focused on developing fine-grained descriptions of their decoding ability and on understanding the relation between their decoding difficulties and three other reading-related domains: phonemic awareness, word identification, and reading comprehension. By characterizing the abilities and limitations of these poor decoders, we hoped to understand better how particular features of explicit phonics activities might lead to benefits in each of these skills. Across a battery of cognitive

\footnotetext{
${ }^{1}$ The term phonics has had a long history of use in reading instruction, but it has also been the subject of much ambiguity and misunderstanding. It implies an emphasis either on phonology (the sound structure of language) or on phonemes (the inventory of sounds in a language), and on acquiring connections between these and letters. Phonic approaches to instruction are highly variable and sometimes incorporate or neglect activities ranging from phonemic awareness training with spoken words, extensive drills in letter-sound correspondences, presentation of words in rhyme families, explicit instruction of rules that capture regularities in letter-sound combinations, implicit structuring of materials, systematic presentation of orthographic patterns that are introduced either implicitly or explicitly, to opportunistic approaches that point out letter-sound correspondences during text reading.
} 
measures, we sought to understand what abilities these children had mastered already and what abilities were particularly deficient.

Furthermore, we sought to characterize decoding difficulties at a fine-grade level by considering decoding performance on each phoneme position within a word form. Children who exhibit decoding difficulties beyond the first grade may demonstrate adequate command of letter sound correspondences for word-initial graphemes but may fail to apply that knowledge to other positions within a word (e.g., to the second letter of an initial consonant cluster or a final consonant in a word) and may have particular difficulty with vowels. To the extent that this is true, such children could be said to have grasped only a limited form of the alphabetic principle in that they do not generalize their grapheme-phoneme knowledge to all the positions within a word form. We examined this possibility by analyzing poor readers' decoding skills as a function of grapheme position within words.

A second goal of our study was to examine the impact of a decoding skills intervention program on this cognitive reading skills profile. For the purpose of this study, we selected a decoding intervention program that contained features that, by our analysis, might be particularly well suited to help children with poor decoding skills make rapid improvements. The activities that form the basis of our intervention were adapted from an instructional approach called Word Building, developed by Isabel Beck (Beck, 1989; Beck \& Hamilton, 2000) and inspired by guidance from effective practice. In the adaptation of Word Building for our study, the materials were developed into a fully scripted series of steps that could be followed by novice tutors with minimal training. In addition, a system of periodic progress exams and progression rules were introduced to modify the difficulty of materials in line with individual students' changing abilities on a continuous basis.

Although informal reports from teachers who have used versions of this program have been generally encouraging, this report represents the first empirical test of Word Building. As the first such investigation, we set out to document the impact of supplementing the ordinary educational activities of our sample of poor decoders with 20 tutoring sessions of Word Building. Children were randomly assigned to either an Intervention group or a Control group. Control group children were enrolled in a 4-month waiting list to receive the Word Building intervention and were encouraged to continue pursuing any form of reading skills support available during this time span.

Measures of reading skill collected before and after the 4-month period of the study included fine-grained analyses of decoding abilities by grapheme position in addition to standardized measures of decoding skill, word identification, phonemic awareness, and reading comprehension. In this way, we were able to directly assess the impact of 20 sessions of Word Building on specific aspects of decoding skills and other reading-related skills. As this intervention program contained in- 
structional activities that are not universally present in most phonics programs, we describe the activities in detail and also provide rationale for why these particular activities might be effective at improving the reading skills of children with poor decoding abilities. However, under the design of this initial study, evidence linking intervention activities and particular outcomes are limited to the whole-program level, and future research will be required to link particular activities within the intervention to particular reading skill improvements and to contrast the effectiveness of this program with other programs.

A core aspect of Word Building is an instructional activity called progressive minimal contrasts, which may be unique among decoding programs, and represents a potentially important contribution to the methods available to teach decoding. This activity provides a chain of words that differ by a single grapheme. A child forms the words in a lesson with letter cards by stepping through a scripted set of transformations that change one word into the next by changing a single grapheme at the beginning, middle, or end of the word. After each transformation, the child decodes the new word, which looks and sounds similar to the previously decoded word. This activity is designed to help children attend to the subtle impact of a single grapheme change on the appearance and pronunciation of each word. Focusing attention on each individual letter sound unit within words may play an important role in developing fully specified representations of printed words.

The idea that the quality of a child's word representations in reading can be improved by focusing attention on every letter within printed words is consistent with theoretical proposals by Perfetti (1991) and Ehri (1991). These proposals emphasize the learner's acquisition of specific visual word forms through connections between orthography and phonology at the sublexical and lexical levels. For example, in the Restricted-Interactive Model (Perfetti, 1991), the key development in learning to read is the acquisition of word representations whose constituent letters and phonemes become increasingly specified in all word positions. In this model, the development of decoding ability concurrently assists in the acquisition of specific words and helps to develop a set of resources for the pronunciation and identification of unfamiliar words.

The notion that decoding ability plays a central role in building word recognition skill was further elaborated by Share and Stanovich (1995). Their self-teaching hypothesis holds that facility in manipulating sublexical letter-sound units provides a child with a reliable means of producing an adequate pronunciation for newly encountered printed words and enhances the quality of print-addressable lexical representations for words the child encounters repeatedly. Thus, as children begin to decode words efficiently on the basis of each available grapheme-phoneme unit, they begin to benefit from their reading experiences in two ways: They strengthen their word decoding abilities, and they build representations of words that are more accurate and more fully specified. 
With an increasing emphasis on the importance of phonemic awareness in learning to read (National Reading Panel, 2000; Snow et al., 1998), we also sought to document the effects of Word Building on phonological awareness. Although Word Building does not directly incorporate many of the activities found in phonological awareness instructional programs, the progressive minimal contrast activity might have an important impact on developing phonological awareness skills by focusing children's attention on units within each position of the written and spoken word. Given the growing consensus of research demonstrating the reciprocal relation between decoding and phonological awareness skills (Perfetti, Beck, Bell, \& Hughes, 1987; Wagner, Torgesen, \& Rashotte, 1999), it is important to investigate how decoding activities might serve to strengthen phonological awareness skills in the process of improving decoding abilities.

On the issue of comprehension, a simplified theoretical position holds that reading is equal to decoding plus general language comprehension. Thus, word reading in combination with general comprehension skill should determine reading comprehension success to a reasonable degree, as Gough and Hoover (1990) and others have argued. In support of this view, a recent analysis of the relation between decoding abilities and reading comprehension abilities demonstrated that these skills are generally tightly correlated during early reading (Shankweiler et al., 1999).

Nevertheless, increasing decoding skills, although arguably central to the development of reading skill, does not necessarily produce transfer benefits into text comprehension skill. For example, a study by Torgesen et al. (1999) showed impressive gains in decoding skills for children who started in the impaired the range but demonstrated a lack of any significant improvements in passage comprehension scores (although, see Torgesen et al., 2001, for a counterexample). Therefore, an important goal for future research is to shed light on conditions that best promote such transfer. For this reason, we investigated the impact of Word Building on passage comprehension measures and highlight some of the features of the program that might successfully encourage such transfer.

Inherent in many phonics approaches is the notion that children should not experience decoding as an exercise unrelated to actual reading but should have the chance to experience immediately the payoff of increasing their decoding repertories by encountering new decodable words within connected text. Thus, integrating the content between decoding activities and text reading activities may be one way to promote transfer of decoding benefits into comprehension benefits. Furthermore, tutor-guided text reading activities may encourage transfer from decoding gains to comprehension gains by helping children orchestrate their efforts both to decode the words accurately and to comprehend message information. The integration of decoding and text reading is an inherent part of the Word Building intervention, and thus this program serves as an interesting test case for examining improvements in reading comprehension measures. 


\section{STUDY OVERVIEW}

To gain a better understanding of the cognitive reading skills of children who are poor decoders, we first selected a group of children who demonstrated poor decoding skills on standardized tests. We then characterized their reading-related skills across a wider array of measures designed to elucidate problems in decoding skills as well as to characterize word recognition, passage comprehension, and phonological awareness skills. Half of the children were randomly assigned to engage in a laboratory-based after-school tutorial program that focused on decoding skills, and the other half of the children joined a waiting-list group to receive the same intervention the following semester. We examined the impact of the intervention on decoding skills via standardized and experimental tests. Finally, we examined the impact of the intervention on the wider array of reading skill measurements.

\section{METHODS}

\section{Participants}

Children between 7 and 10 years of age who had completed at least the first grade yet still demonstrated reading difficulties were recruited from elementary schools in a large metropolitan area. Instructional support teachers distributed a flier to parents describing an intervention study for children experiencing reading difficulties such as sounding out new words and reading grade-level material. Telephone interviews were conducted with parents to explain the screening process and to determine whether their child might qualify for the study. Thirty-eight children who passed the selection process (see Selection Procedure in the following discussion) volunteered with parental consent to return for additional assessments and enter a 4-month study. ${ }^{2}$ Intervention and control groups were formed via an iterative random assignment procedure that assured the groups were well matched for age, sex, and performance on the pretest measures. Group assignment was conducted with the sample of 38 children who had initially volunteered for the study. Subsequently the two groups suffered substantial attrition over the 4 months of the study, largely due to the inconvenience caused to parents who had to transport children to the study location. This created a potential source of group differences. Fortunately the two remaining groups ( 12 in each) were well matched for age, for sex ( 7 boys and 5 girls in each group), and on the entire array of pretest measures. All group compari-

\footnotetext{
${ }^{2}$ During the time span of this study, many of these children were recruited for other experiments not directly related to the issues of this article. These experiments included functional magnetic resonance imaging (fMRI) measures, naming speed measures, and auditory processing of tones and spoken words, and they are reported elsewhere.
} 
sons resulted in $t$ values less than 1, with the exception of the Elision task (Wagner, Torgesen, \& Rashotte, 1999), in which a nonsignificant trend suggested that the control group may have performed slightly better, Elision $z$ scores, $t(23)=1.26, p<$ .28. To facilitate clear comparisons between our pretest analysis of the cognitive skills profile and the comparisons of changes in skill before and after the intervention all results reported in the following discussion are restricted to the 24 children who completed the study.

\section{Selection Procedures}

A screening battery consisting of standardized tests and parental questionnaires was designed to select children who demonstrated decoding difficulties yet also showed roughly normal language abilities, as indexed by standardized tests of receptive oral vocabulary. Furthermore, the study was restricted to children who demonstrated no evidence of clinical-range severity of symptoms that might indicate other neurological or psychological disorders (e.g., attention deficit disorder/attention deficit hyperactivity disorder, anxiety of depressive disorders). These additional restrictions were motivated by the fact that these children were also being recruited for a related fMRI study that stipulated such restrictions and that the decoding intervention protocol was to be administered by undergraduate volunteers with little or no training in working with children from special populations.

Receptive oral vocabulary was assessed with the Peabody Picture Vocabulary Test-Revised (PPVT-R; Dunn \& Dunn, 1981). The inclusion criterion was a standard score greater than 85. Parents completed the Child Behavior Checklist (CBCL; Achenbach \& Edelbrock, 1983); the Conner's Parent Rating Scale (Conner, Sitarenios, Parker, \& Epstein, 1998); and a questionnaire concerning professional diagnoses of psychological, neurological, visual, and auditory impairments. Children with either a physician's diagnosis or indication of such disorders in the clinical range ( $>95$ th percentile) were excluded. Decoding ability was assessed with the Woodcock Reading Mastery Test-Revised (WRMT-R; Woodcock, 1998) Word Attack subscale. This test presents children with increasingly complex novel pseudowords. Half the participants received Form $\mathrm{G}$ and the other half Form $\mathrm{H}$.

The 50 children with the poorest decoding skills who passed the other exclusion criteria were invited into the study. Thirty-eight agreed to participate, and 24 completed the study. Of these 24, 20 fell below the 30th percentile on Word Attack, the level recommended by Torgesen (in press) as a working definition of reading impairment, and all 24 fell below the 40th percentile. Use of this more liberal criteria holds the advantage of providing case study information on the effects of this previously untested program on a wider range of decoding abilities, potentially re- 
vealing a relation between severity of decoding difficulties and outcome. However, this also introduces difficulties when trying to compare results to studies that use more stringent inclusion criterion.

\section{Additional Measures of Reading-Related Skill}

Word identification. The Word Identification subscale of the WRMT-R (Forms $\mathrm{G}$ and $\mathrm{H}$ ) was also administered during the initial visit to characterize children's abilities to read relatively high frequency words, many of which are not decodable at the level of grapheme-phoneme constituents.

Reading comprehension. The WRMT-R Passage Comprehension subscale (Forms $\mathrm{G}$ and $\mathrm{H}$, counterbalanced across participants) requires children to read short texts ranging from single sentences to complex paragraphs and respond to each by filling in a blank embedded in the text.

Phonemic awareness. Blending Words, Blending Nonwords, and Elision subtests were administered from a prototype version of the Comprehensive Test of Phonological Processing (Wagner et al., 1999). In the Blending Words test, children listened to segments of words on a tape recorder and were asked to blend them together into a word pronunciation. The Blending Nonwords test followed similar procedures, except that the segments combined to form pronounceable pseudowords. In the Elision test, the experimenter read a word and then asked the child what real word would be made if a particular phoneme was removed. (e.g., "Say brake; now say brake without saying /r/"). The Initial Sound Matching test presents children with a spoken sample and a corresponding picture (e.g., sun), followed by three additional spoken words with corresponding pictures. The child's task is to choose the picture that starts with the same sound as the sample. The Final Sound Matching test is identical, except that the decision is made about the final consonant. Note that the Sound Matching tests were originally designed and standardized for use with children in kindergarten and first grade, thus only raw scores (percent correct) are reported.

Experimental pseudoword tests. Children were asked to read aloud a list of 128 monosyllabic pseudowords presented one at a time on a computer screen. Pseudowords were constructed by sampling words from the Word Building intervention and recombining onset and rime units to form novel pseudowords. Experimenters transcribed each response after each trial by attempting to spell the child's 
pronunciation in pseudophonetic form. ${ }^{3}$ Two analyses were conducted on the written responses. The first analysis scored pronunciation accuracy separately for the onset, nucleus (vowel), and coda regions of each pseudoword. Finer grained analyses investigated the influence of position on decoding consonants in the onset and coda regions by scoring accuracy for each individual consonant. For example, 96 of the onsets contained a single consonant (e.g., gip), and 32 contained clusters of two consonants (e.g., glom). Within separate analyses of onset and coda regions, accuracy was scored separately for single consonants $(n=96)$, consonants that appeared in the initial position of a cluster $(n=32)$, and consonants that appeared in the final position of a cluster $(n=32)$.

\section{Intervention Materials}

Word Building materials used in this study were adapted from Beck and Hamilton $(1996,2000)$. The intervention program consisted of 77 lessons. Each lesson included a set of 5 to 16 letter cards, instructions on how to form a chain of words that differed by a single letter transformation, (as shown in Figure 1), a set of flashcards containing each word formed in the lesson, and a set of sentences consisting mostly of words formed in the lesson. Lessons were grouped into 23 units that each contained 3 to 5 lessons preceded and followed by a short test.

The lessons progressively incorporated more difficult grapheme-phoneme units and word forms (e.g., from consonant-vowel-consonant [CVC] to CCCVCC). The first 10 units focused on short vowels; the next 5 introduced long vowel sounds controlled by silent $e$; the next 4 units dealt with vowel digraphs (e.g., ee, ai, oa, ow, oy); and the final 4 units involved changes in vowel sounds in different phonetic environments (i.e., changes in vowel pronunciation when a vowel is followed by an $r$ ).

Within each lesson, children were given a small set of letter cards from which they built words, as directed by the tutor. After the child formed a word and successfully read it aloud, the tutor instructed the child to insert, delete, or exchange a

\footnotetext{
${ }^{3}$ The reliability of the online transcription protocol has not been rigorously evaluated, and such evaluation is no longer possible, given that responses were not tape-recorded. Experimenters ranged in their level of training from holding a doctoral degree in the field of psycholinguistics to undergraduates with approximately $30 \mathrm{~min}$ of explicit training in the transcription protocol. Nonetheless, three facets of the experiment minimized the potential impact of transcription errors. First, the experimenter was able to view each target pseudoword, and thus the proper transcription of a correct response was modeled on the screen. Second, advancement from one trial to the next was controlled by the experimenters, thus each new trial began only after the experimenters were confident they had correctly heard and transcribed the child's response on the current trial. Finally, all transcription data was converted into discrete binary values (correct $=1$, incorrect $=0$ ) for each grapheme-phoneme position, thus transcription errors of mispronounced phonemes would have minimal impact on the analyses presented.
} 

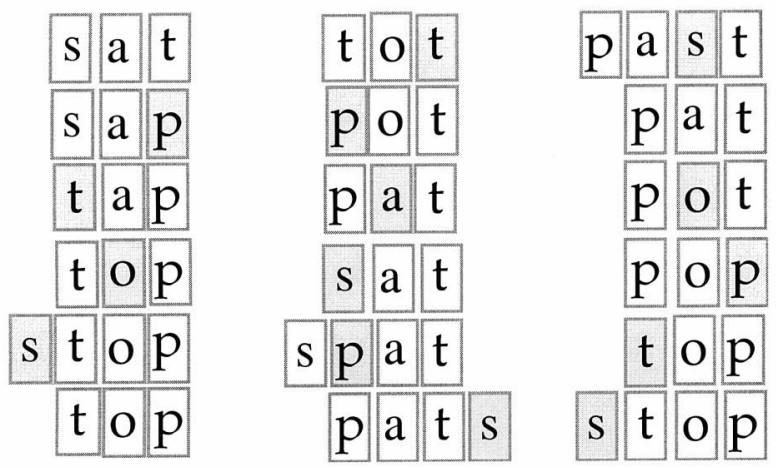

FIGURE 1 Illustration of the progression of word transformations in the word decoding activity of the Word Building intervention. After creating an initial word from letter cards, children are given instructions to change a particular letter card (e.g., take away $t$ and put $p$ in its place) and then to read the newly formed word. The new grapheme card in each trial is highlighted in gray to illustrate how each word transformation focuses a child's attention on different positions in the word form by holding constant the other letters from the previous word.

specific letter card, which would transform the current word into the next word in the lesson sequence. Figure 1 displays an example of a chain of single letter transformations in a typical lesson.

The sequences of letter changes were developed so that, when possible, attention is drawn to each position within a word form (i.e., to the initial consonant, final consonant, vowel, initial, or second consonant within an onset or coda cluster). Furthermore, the letter changes were designed, to the extent possible, to ensure that the same letters that appear in the initial position of words also appear in other positions.

\section{Intervention Procedures}

Children in the intervention group participated in 20 tutorial sessions lasting approximately $50 \mathrm{~min}$ each, as frequently as 3 sessions per week, and completed 4 additional sessions for testing purposes between July and October.

Each child was paired with an undergraduate tutor who participated in the study for credit or for an hourly wage. Most tutor-student pairs remained constant during the intervention. All tutoring took place in a separate quiet room as parents or guardians remained in a nearby waiting area. The laboratory reading center was located on the campus of a large university in a large city in the northeastern United States. Participants in the control group were given educational materials from the 
America Reads Program, and their parents were encouraged to seek any reading support they wanted while their child was on the waiting list to receive the Word Building intervention the following semester.

In the current adaptation of Word Building, the intervention was scripted into step-by-step instructions and decision-making algorithms that could be administered with minimal training by college undergraduates. As previously noted, at the beginning of a lesson, tutors presented each child with several letter cards to be used during that lesson and reviewed their associated sounds. Then the tutor instructed the child to "build" words as illustrated in Figure 1.

Throughout all of the activities, if children had difficulty decoding a word, the tutors avoided pronouncing the word and instead helped scaffold the children's decoding attempts through a small repertoire of scripted activities. When children failed to make an attempt at pronouncing a word, tutors encouraged an attempt based on the letter sounds. When children struggled to combine letter sounds, tutors guided them through the process of progressively blending the sounds together. When children mistook a word for a similarly spelled word, tutors wrote out both the target word and the erroneous word and encouraged the children to analyze the differences between the two words.

After completing a Word Building sequence (i.e., the chain of words in a sequence of minimal contrast transformations), the tutor administered a brief flashcard assessment to determine whether the child could read at least $80 \%$ of the lesson words correctly. If the accuracy criterion was not met, more Word Building activities were carried out using the same words.

Each lesson concluded with a sentence reading activity that contained a high proportion of words that had just been decoded and others that could be decoded given the material the child had covered thus far. Some nondecodable words (i.e., words that could not be decoded based on the child's decoding repertoire) were included. For all decodable words, the tutor encouraged the child to decode the word and, as needed, provided help in the form of scaffolding activities noted previously. If necessary, tutors pronounced nondecodable words. Sentences usually took the form of silly questions (e.g., "Can a sick kid kick a stick?"). After the child successfully read a sentence, the tutor and child engaged in a playful discussion about the meaning of the sentence.

Children progressed through the materials at their own pace. At the beginning of each unit, they were given a pretest of words sampled from the unit lessons. If decoding accuracy was $90 \%$ or greater, the child was permitted to skip that unit and progress to the next unit pretest. If accuracy was less than $90 \%$ on a unit pretest, the child was given the lessons of that unit. On completion of the lessons within a unit, a unit posttest was administered. The same $90 \%$ accuracy criterion was used to determine if the child could progress to the next unit pretest or needed more time on the current unit. These promotion and retention algorithms func- 
tioned continuously to adapt the difficulty level of materials to the child's emerging abilities.

Posttest measures. The same battery of reading skill measures used in the pretest were administered a second time at the completion of the intervention, with the exception of the PPVT-R and parent questionnaires. The two alternate forms of the WRMT-R (Forms $\mathrm{G}$ and $\mathrm{H}$ ) were counterbalanced across pretest and posttest for individual participants.

\section{RESULTS AND DISCUSSION}

\section{Pretest Measures}

Table 1 displays both the means and standard deviations for participant age, decoding ability, and oral vocabulary used in the selection process and the pretest results for the two additional standardized tests of passage comprehension and phonological processing that were not used in the selection process.

Experimental pseudoword pretest. As reported in Table 2, examination of decoding accuracy within different regions of a word form demonstrates that decoding attempts were relatively accurate within the onset region, worse within the coda region, and worse still within the vowel region. An analysis of variance (ANOVA) demonstrated a significant main effect of region, $F(2,44)=62.84, p<$ .0001 , and planned comparisons demonstrated that accuracy within the onset region was superior to the vowel region, $t(22)=125.49, p<.0001$, and also superior to the coda regions, $t(22)=35.81, p<.0001$. Furthermore, accuracy within the vowel region was significantly lower than accuracy within the coda region, $t(22)=$ $27.24, p<.0001$. Although the factor of group (intervention, control) was entered in each of the analyses reported, no main effects or interactions containing the group factor reached significance for the experimental pseudoword pretest.

A post hoc analysis was conducted to rule out the possibility that observed position effects for consonants were a result of more difficult consonants appearing in coda versus onset regions. A subset of materials was selected such that the same consonants appeared equally often within the onset and coda regions. This subset included 54 single-consonant onsets (out of 96) and 54 single-consonant codas (out of 96). Accuracy scores within this subset of materials revealed superior consonant decoding accuracy within onset relative to coda regions, as demonstrated by a significant main effect of region, $F(1,22)=54.06, p<.0001$. 
TABLE 1

Sample Size, Mean, and Standard Deviation for the Selection Measures and Additional Standardized Pretest Measures

\begin{tabular}{|c|c|c|c|}
\hline & $n$ & $M$ & $S D$ \\
\hline \multicolumn{4}{|l|}{ Selection measures } \\
\hline Age (in months) & 24 & 100.5 & 11.6 \\
\hline \multicolumn{4}{|l|}{ WRMT-R Word Attack } \\
\hline Raw score & 24 & 6.6 & 4.6 \\
\hline Percentile rank & 24 & 21.7 & 9.9 \\
\hline Standard score & 24 & 87.5 & 5.5 \\
\hline \multicolumn{4}{|l|}{ WRMT-R Word Identification } \\
\hline Raw score & 24 & 33.5 & 12.4 \\
\hline Percentile rank & 24 & 27.8 & 17.7 \\
\hline Standard score & 24 & 90.0 & 8.6 \\
\hline PPVT-R (raw) & 24 & 119.0 & 17.4 \\
\hline PPVT-R (standard score) & 24 & 105.0 & 10.9 \\
\hline \multicolumn{4}{|c|}{ Additional measures } \\
\hline \multicolumn{4}{|c|}{ WRMT-R Passage Comprehension } \\
\hline Raw scores & 23 & 15.9 & 8.0 \\
\hline Percentile rank & 23 & 27.0 & 14.6 \\
\hline Standard score & 23 & 89.2 & 9.0 \\
\hline \multicolumn{4}{|l|}{ СТPР } \\
\hline \multicolumn{4}{|l|}{ Word Blending } \\
\hline Raw score & 24 & 12.0 & 3.31 \\
\hline$z$ score & 24 & -0.86 & 0.59 \\
\hline \multicolumn{4}{|l|}{ Nonword blending } \\
\hline Raw score & 24 & 9.7 & 3.5 \\
\hline$z$ score & 24 & -0.55 & 0.75 \\
\hline \multicolumn{4}{|l|}{ Elision } \\
\hline Raw score & 24 & 11.91 & 4.5 \\
\hline$z$ score & 24 & -1.25 & 0.75 \\
\hline Initial sound matching $(\%)$ & 24 & 83.5 & 21.5 \\
\hline Final sound matching $(\%)$ & 24 & 53.6 & 29.8 \\
\hline
\end{tabular}

Note. $\quad$ WRMT $-\mathrm{R}=$ Woodcock Reading Mastery Test-Revised; PPVT $-\mathrm{R}=$ Peabody Picture Vocabulary Test-Revised; CTPP $=$ Comprehensive Test of Phonological Processing. ${ }^{4}$

Position analyses within the onset region. Decoding accuracy within the onset region is further broken down in the second section of Table 2, revealing a pattern of high accuracy for all word-initial consonants and lower accuracy for consonants in the second position of an onset cluster. ANOVA results revealed a significant effect of position, $F(2,44)=31.0, p<.0001$. Planned comparisons revealed

\footnotetext{
${ }^{4}$ Note that as these data were collected on an experimental version of the Comprehensive Test of Phonological Processing (Wagner et al., 1999), The reported $z$ scores do not reflect on the national standardization sample used for the published version of this instrument but rather reflect an early standardization sample consisting of 100 children at each grade level.
} 
TABLE 2

Mean Accuracy (Percent Correct and Standard Deviations) of Decoding Attempts on Individual Segments of Experimental Pseudoword Test

\begin{tabular}{lcc}
\hline Variable & $M$ & $S D$ \\
\hline Regions within word-form (\% correct) & & \\
$\quad$ Onset & 70.1 & 15.2 \\
$\quad$ Vowel & 40.0 & 18.6 \\
$\quad$ Coda & 54.1 & 20.2 \\
Individual consonants within onset (\% correct) & & \\
$\quad$ Singular & 79.1 & 10.9 \\
Initial position in cluster & 84.3 & 14.0 \\
Second position in cluster & 51.2 & 32.3 \\
Individual consonants within coda (\% correct) & & \\
$\quad$ Singular & 58.4 & 19.2 \\
Initial position in cluster & 43.7 & 28.7 \\
Second position in cluster & 60.3 & 21.6 \\
\hline
\end{tabular}

Note. Segment accuracy is calculated separately for region (onset, vowel, coda) and individual consonant positions. $n=24$.

that performance on the second position within an onset consonant cluster was significantly less accurate than for onset consonants that appeared either singularly, $t(23)=38.1, p<.0001$, or within the first position of an onset cluster, $t(23)=53.6, p$ $<.0001$.

Post hoc comparisons on a subset of 24 consonants that appeared equally often as either a singular consonant onset or the initial position of an onset cluster revealed no significant differences in accuracy score. A similar post hoc comparison showed that decoding accuracy was significantly lower for a subset of 14 consonants that appeared in the second position of an onset cluster than when those same 14 consonants occurred as a single consonant onset, $t(23)=25.3, p<.0001 .^{5}$

Position analyses within the coda region. The bottom section of Table 2 displays accuracy for consonants within different positions of the coda region. Unlike the pattern of position effects that appeared in the onset region, position effects within the coda region were characterized by superior performance for the final letter in a word. ANOVA results for the three classes of coda consonant positions (singular consonant codas, the first position, and the second position within consonant clusters in the coda) indicated a significant effect of position, $F(2,44)=15.4, p<$

\footnotetext{
${ }^{5}$ The number of consonants that appeared equally often in the first and second position within an onset cluster ( $n=4$ instances) did not permit post hoc comparison across identical consonants for these two positions.
} 
.0001. Planned comparisons revealed that performance on the first position within a coda consonant cluster was significantly less accurate than for either single coda consonants, $t(23)=20.2, p<.0001$, or consonants in the second position of a coda cluster, $t(23)=25.7, p<.0001$. Furthermore, no significant differences were found between single consonants and consonants in the second position of a cluster.

Again, post hoc comparisons were conducted on subsets of the materials to rule out the possibility that these effects were a result of more difficult consonants appearing in the first position of coda consonant clusters. A comparison based on 21 consonants that appeared equally often in the singular consonant codas and the first position within coda consonant clusters revealed significantly lower accuracy for the first position within coda consonant clusters, $t(23)=4.4, p<.05$. A second analysis based on 22 consonants that appeared equally often in single consonant codas and in the second position of clusters revealed a nonsignificant trend, $t(23)=$ $2.9, p<.10$, toward higher accuracy for single consonant codas.

Passage comprehension pretest. Although Passage Comprehension abilities had no influence on the selection process, children demonstrated poor performance on this measure, as evidenced by a group average near the 27 th percentile. As indicated by the second column in Table 1, unlike the other measures reported, Passage Comprehension data reflects the exclusion of a single participant's pretest and posttest data, based on the observation that this participant's pretest standard score exceeded an outlier exclusion threshold of $3 S D$ s above the group mean.

Phonemic awareness pretest. Standard scores for phonemic awareness measures revealed impairments in performance. To compare relative abilities across the Blending Words, Blending Nonwords, and Elision tests, an ANOVA was conducted on $z$ scores for these three tests (see footnote 4). A significant effect of test, $F(2,63)=3.76, p<.05$, revealed that children were not equally deficient across all three tasks. Planned contrasts showed no significant differences between the two blending tasks but revealed that Elision test performance was significantly more impaired than combined performance on the blending tests, $t(18)=6.751, p<$ .01 . This result suggests that although children selected for decoding difficulties may be somewhat impaired on blending skills, impairments are more pronounced for phonological measures that require the active manipulation of phoneme content within words.

The final two rows in Table 1 display mean accuracy performance on the Sound Matching test for initial consonants versus final single consonants for spoken words. Results demonstrate that, although accuracy on initial consonants was high, matching performance on final phonemes was relatively low, as revealed by 
a significant main effect of position, $F(1,22)=37.19, p<.0001$. The direction of this effect for matching letter sounds bears an interesting similarity to the effects of position found in the experimental pseudoword reading task, suggesting a potentially interesting relation between difficulties in matching sounds and decoding consonants in the word-final position.

\section{SUMMARY OF PRETEST RESULTS}

The results of pretest analyses reveal an overall reading skills profile that documents the children's deficits not only in decoding proficiency for which they were selected, but also in word identification, phonemic awareness, and reading comprehension. The experimental pseudoword test provides some insights into the nature of these decoding deficits. The children appear to have mastered some aspect of the alphabetic principle, as indicated by their relative skill at decoding the first letter of each pseudoword, but they also show consistent patterns of difficulty in decoding other letter positions within word forms. The poorest performance was displayed for letters in the medial position, which in the first analysis consisted entirely of vowels. Although our materials did not allow us to examine interactions between position effects and phoneme class (consonants vs. vowels), it is worth noting that the poor performance on vowels is at least consistent with the observation that the vowel system carries the bulk of the variable mappings difficulties of English, as well as the bulk of reading errors for normal adult readers (Fowler, Liberman, \& Shankweiler, 1977).

Although accuracy for decoding consonants was also deficient, the position analyses suggest that these deficiencies were dependent on where the consonants appeared within a word form. Initial consonants elicited successful grapheme-phoneme translation performance, whereas consonants in other positions elicited less successful performance. One way to describe this tendency is to say that these children have grasped a limited form of the alphabetic principle but do not yet apply it to all positions in a word. In the terms of Ehri's (1999) account of decoding development, these children could be said to have progressed beyond the "pre-alphabetic phase" and into the "partial alphabetic phase," but to have failed to break into the "full alphabetic phase" marked by decoding all letter positions within a word. Alternatively, incremental theories that avoid postulating discrete phases (e.g., Perfetti, 1991; Share \& Stanovich, 1995) might characterize these children's difficulties on a continuum that describes their tendencies to successfully employ letter sound correspondence knowledge during reading.

The Sound Matching results demonstrated a pattern of position effects that were consistent with the decoding results. That is, the children seem to have mastered the phonological skills required to identify and match a single consonant in the initial position of a word, yet they experienced difficulty when the same skill 
was assessed for simple consonants at the ends of spoken words. Furthermore, although these children demonstrated only moderate deficits in blending skills, they were more severely impaired on tasks that required manipulation of phonemes within a word, as in the Elision task.

These pretest findings suggest that children with these difficulties might benefit from activities that target the manipulation of single graphemes and phonemes in various positions within a word. We propose that Word Building activities do this by transforming one word into another through the manipulation of a single letter. Such activities may simultaneously draw attention to graphemic units within printed words and the corresponding phonemic units within spoken words. As such, Word Building may promote gains in decoding and phonemic awareness in tandem.

\section{Progression Through the Intervention Materials}

Participants in the intervention group completed the 20 intervention visits within 14.2 weeks on average $(S D=3.3$, range $=8.9-18.9)$. Over the 20 intervention visits, children in the intervention group completed an average of 32.3 lessons ( $S D=5.6$, range $=23-42$ ). Performance on unit tests determined whether particular lesson materials were assigned, skipped, or repeated. Thus, each child progressed through the 77 sets of increasingly difficult lesson materials in a unique fashion. On average, by the end of the 20th session children completed the materials from the 43rd lesson set $(S D=15.8)$. Two children completed the materials from the 77 th lesson set, and the child who progressed the least completed the materials from the 26th lesson set.

\section{Effect of the Intervention on Reading Skills}

Each set of measures was subjected to a repeated measures ANOVA contrasting group (intervention vs. control) as a between-subjects factor and time span (July pretest vs. October posttest) as a within-subjects factor. Effects that are specifically related to participation in the intervention are evident in terms of Group $\times$ Time Span interactions. In addition, the standardization information of the WRMT-R subscales provided a means of quantifying the number of children in each group who demonstrated significant improvements over the 4-month intervention period by allowing raw scores to be converted into grade equivalence scores. We developed an a priori learning criterion that enabled us to determine whether each child advanced beyond what would be expected of a typical child over a similar time span. Because the intervention spanned a period of less than 4 months, children who demonstrated improvements exceeding one third of a grade level were classi- 
fied as passing the learning criterion. Nonparametric (chi-square) analyses were used to compare the proportion of children in each group who passed the learning criterion. ${ }^{6}$ The chi-square analyses are presented as a complement to the ANOVAs because they place less emphasis on the magnitude achieved by a minority of children and greater emphasis on the proportion of children who achieved a desirable outcome. Two participants in the control group completed many of the posttest measures but did not return to complete their phonemic awareness posttest session. Thus these two participants were excluded from the phonemic awareness analyses.

Experimental pseudoword test. We assessed improvements in decoding accuracy for different positions within a word form by examining accuracy changes in the experimental pseudoword reading test. Figure 2 demonstrates changes in decoding accuracy in the onset, nucleus (vowel), and coda regions over the course of the intervention time span.

Participants in the intervention group showed larger decoding improvements than participants in the control group in all three regions of word forms, leading to a significant Group $\times$ Time span effect, $F(1,22)=17.1, p<.0005$. These results demonstrated that the intervention was successful at improving decoding abilities in the onset, vowel, and coda regions of the word form. A significant Region $\times$ Group $\times$ Time interaction, $F(2,44)=6.0, p<.005$, indicated that the intervention group improved more rapidly for vowel and coda regions than for onset regions. However, any interpretation of this result must bear in mind the fact that pretest performance for onset regions was much closer to the upper limit of $100 \%$ accuracy than performance in the other position conditions.

Figure 3 demonstrates changes in decoding performance in three consonant positions (i.e., singular, initial position in a cluster, second position within a cluster) that occurred in the onset and coda regions.

Results within the onset region showed that whereas difficulties in decoding the second position in an onset cluster persisted for the waiting-list control group, the intervention group demonstrated dramatic gains in this position. This observation was supported by a significant Position $\times$ Time $\times$ Group interaction, $F(2,44)=8.1$, $p<.001$. Results within the coda region indicate that the difficulties in decoding consonants persisted in the waiting-list control group, including less accurate decoding of the first consonant of a coda consonant cluster. By contrast, the intervention group demonstrated improvements in all three coda positions, leading to a significant Time $\times$ Group interaction, $F(1,22)=8.7, p<.01$.

Taken together, the various analyses of position effects on decoding accuracy converge to indicate that deficient decoding abilities for grapheme positions be-

\footnotetext{
${ }^{6}$ A similar chi-square analysis was conducted in which children were classified as either gaining or losing in terms of standard scores. Both approaches produced the same pattern of results.
} 


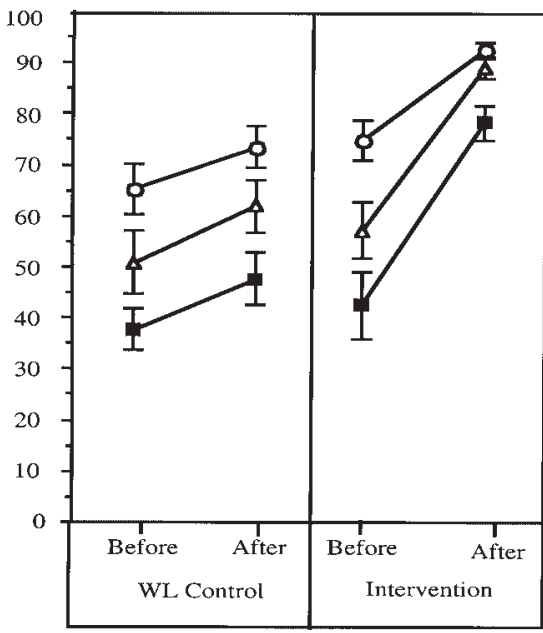

- Onset

- Coda

- Vowel

FIGURE 2 Mean accuracy (with standard error bars) for the Pseudoword Reading Task before and after the intervention, as a function of region within each pseudoword (Onset, Vowel, Coda).

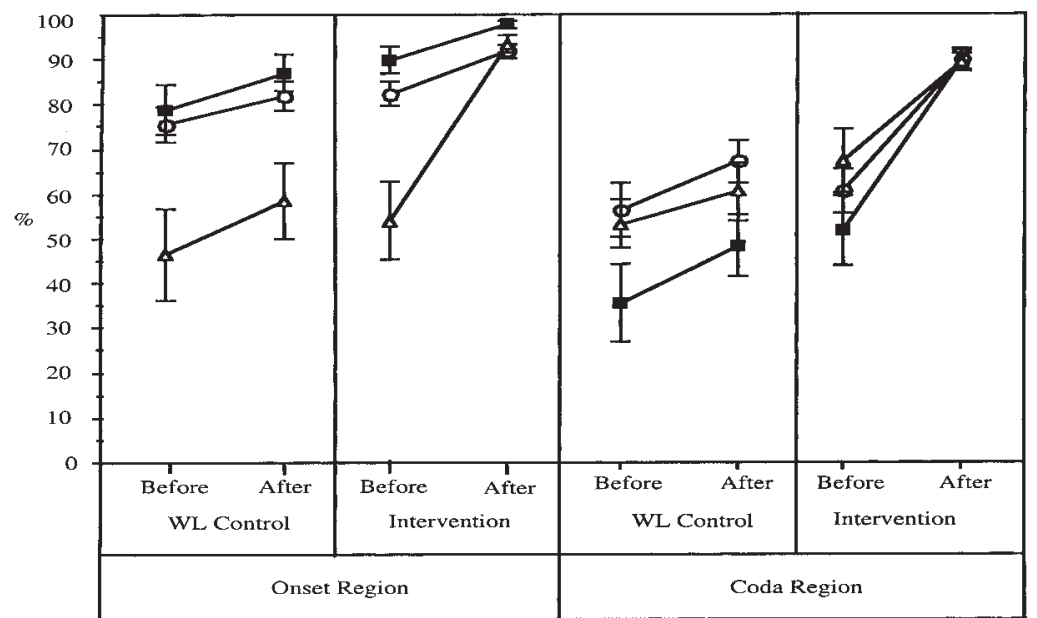

- Singular $\square$ Ist in Cluster $\Delta$ 2nd in Cluster

FIGURE 3 Mean accuracy (with standard error bars) for performance within the onset region of the pseudoword task before and after the intervention as a function of phoneme position (Singular, First in Cluster, Second in Cluster). 
yond the initial grapheme tended to persist over time in the absence of intervention and that the intervention led to improvements in the application of decoding skills within each position of a word form.

Word attack (decoding). Changes in decoding skills were assessed by a repeated measures ANOVA of the WRMT-R Word Attack subscale. As Table 3 demonstrates, the intervention group displayed larger improvements in Word Attack scores than the control group. ANOVA results revealed a main effect of time span that was significant in the analysis of raw scores, $F(1,22)=24.8, p<.0001$. A main effect of group was revealed to be significant for standard scores, $F(1,22)=$ $4.3, p<.05$. Most important for our purposes, the Group $\times$ Time Span interaction

TABLE 3

Pretests and Posttests Means and Standard Deviations for Raw Scores, Standard Scores, and Grade Equivalent Scores from the Word Attack, Word Identifification and Passage Comprehension Subscales of the Woodcock Reading Mastery Test-Revised

\begin{tabular}{|c|c|c|c|c|c|c|c|c|c|}
\hline \multirow[b]{3}{*}{ Group } & \multicolumn{9}{|c|}{ Measure } \\
\hline & \multicolumn{3}{|c|}{ Raw Scores } & \multicolumn{3}{|c|}{ Grade Equivalency } & \multicolumn{3}{|c|}{ Standard Scores } \\
\hline & Pretest & Posttest & Gain & Pretest & Posttest & Gain & Pretest & Posttest & Gain \\
\hline \multicolumn{10}{|c|}{ Word Attack } \\
\hline \multicolumn{10}{|c|}{ Control $^{\mathrm{a}}$} \\
\hline$M$ & 6.4 & 7.3 & 0.9 & 1.5 & 1.7 & 0.2 & 87.7 & 87.0 & -0.7 \\
\hline$S D$ & 5.1 & 6.9 & & 0.9 & 1.0 & & 6.1 & 10.0 & \\
\hline \multicolumn{10}{|c|}{ Intervention $^{\mathrm{a}}$} \\
\hline$M$ & 6.8 & 14.8 & 8.0 & 1.6 & 2.8 & 1.2 & 87.3 & 94.0 & 6.8 \\
\hline$S D$ & 4.3 & 5.4 & & 0.4 & 0.8 & & 5.2 & 4.6 & \\
\hline \multicolumn{10}{|l|}{ Word } \\
\hline \multicolumn{10}{|c|}{ Identification } \\
\hline \multicolumn{10}{|c|}{ Control $^{\mathrm{a}}$} \\
\hline$M$ & 31.4 & 31.6 & 0.2 & 1.9 & 2.0 & 0.1 & 90.4 & 86.8 & -3.6 \\
\hline$S D$ & 14.7 & 14.5 & & 0.5 & 0.4 & & 10.1 & 7.1 & \\
\hline \multicolumn{10}{|c|}{ Intervention $^{\mathrm{a}}$} \\
\hline$M$ & 35.5 & 38.8 & 3.3 & 2.1 & 2.3 & 0.2 & 89.6 & 88.4 & -1.2 \\
\hline$S D$ & 9.9 & 10.2 & & 0.3 & 0.5 & & 7.3 & 9.0 & \\
\hline \multicolumn{10}{|c|}{ Passage } \\
\hline \multicolumn{10}{|c|}{ Comprehension } \\
\hline \multicolumn{10}{|c|}{ Control $^{\mathrm{a}}$} \\
\hline$M$ & 15.4 & 17.6 & 2.2 & 1.7 & 2.0 & 0.3 & 90.7 & 89.3 & -1.3 \\
\hline$S D$ & 7.5 & 9.8 & & 0.7 & 1.2 & & 7.6 & 9.2 & \\
\hline \multicolumn{10}{|c|}{ Intervention ${ }^{\mathrm{b}}$} \\
\hline$M$ & 16.6 & 24.3 & 7.7 & 1.9 & 2.7 & 0.8 & 87.6 & 93.9 & 6.3 \\
\hline$S D$ & 9.5 & 7.8 & & 0.8 & 1.1 & & 10.5 & 8.8 & \\
\hline
\end{tabular}

${ }^{\mathrm{a}} n=12 .{ }^{\mathrm{b}} n=11$. 

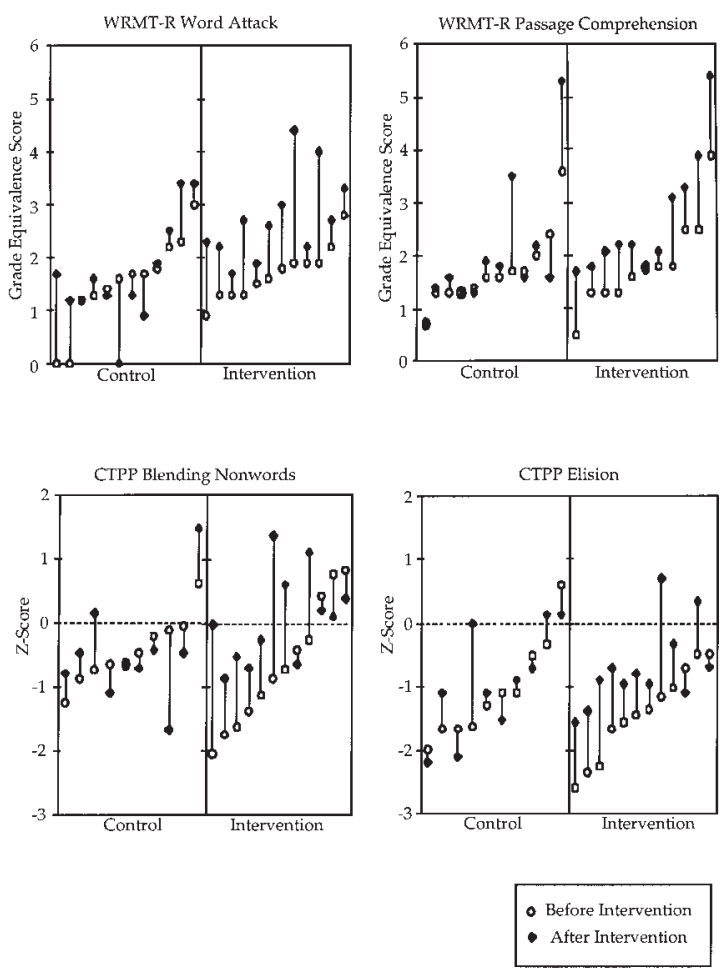

FIGURE 4 Individual performance scores before and after the intervention for the four subscales demonstrating significant Group $\times$ Time Span Interactions. Scores of individuals within each group are arranged from right to left in rank order of pretest performance. Passage Comprehension results are presented in Grade Equivalence scores. Comprehensive Test of Phonological Processing (CTPP) Nonword Blending and Elision results are presented as $z$ scores.

was demonstrated to be significant in both the analysis of raw scores, $F(1,22)=$ $15.7, p<.0007$, and in the analysis of standard scores, $F(1,22)=5.243, p<.032$.

The upper left panel of Figure 4 illustrates that participation in the intervention group led to gains for all participants, ranging from nominal improvements to improvements of several grade levels, whereas the control group demonstrated fewer improvements over the same time span.

The learning criterion analysis (described previously) demonstrated that 11 of the 12 children in the intervention group produced gains that surpassed the one third grade level learning criterion. During that same time only 4 of the 12 children in the control group passed this criterion. Chi-square comparison demonstrated that the proportion of children in the intervention group who surpassed the criterion was significantly greater than in the control group, $\chi^{2}(4, N=24)=8.71, p<.01$. 
Word identification. As indicated in Table 3, gains in Word Identification raw score were minimal, and standard scores actually document a slight decrement in performance over time relative to the sample of children used to construct the norms. ANOVA results for raw scores revealed no significant effects for group, time span, or a Group $\times$ Time Span interaction. However, analyses of standard scores revealed a significant main effect of group, $F(1,22)=1.688, p<.014$, suggesting that the intervention group slightly outperformed the control group when overall performance is pooled across pretest and posttest measures. Furthermore, a main effect of time span that approached significance, $F(1,22)=3.374, p<.08$, suggested that although raw scores remained relatively stable over time, children in both groups may be falling behind the normative sample in their development of Word Identification skills over the tested time interval. Standard score analysis revealed no significant Group $\times$ Time Span interaction, $F(1,22)=0.873, p<.3601$, and thus provided no evidence to suggest that the intervention impacted this measure. Furthermore, a chi-square analysis of the proportion of children in each group passing the learning criterion did not approach significance, $\chi^{2}(4, N=24)=0.38$.

Results of the Word Identification test stand in stark contrast to the results of both the experimental Pseudoword test and the Word Attack subscale of the WRMT-R. One explanation for this discrepancy is that the Word Identification test contains a high proportion of irregular or inconsistent words that cannot necessarily be read more accurately by enhancing grapheme-phoneme decoding skills. Of the 126 test words from Forms $\mathrm{G}$ and $\mathrm{H}$ of the Word Identification test that fell within the range of improvements for the children in this study, approximately $60 \%$ of these items were nondecodable using the grapheme-phoneme elements trained in the 77 lessons of the intervention. ${ }^{7}$ This suggests that the items on the Word Identification test are not necessarily sensitive to gains in decoding words with regular pronunciations.

Passage comprehension. As summarized in Table 3, the intervention group produced larger Passage Comprehension gains over the time span of the study than did the control group. ANOVA results revealed a main effect of time span that reached significance for raw scores, $F(1,21)=22.8, p<.0001$, but not standard scores, $F(1,21)=1.967, p<.1754$. Both raw and standard scores revealed a significant Group $\times$ Time Span interaction, $F(1,21)=6.2, p<.02$ and $F(1,21)=$ $4.664, p<.04$, respectively, demonstrating that the intervention group accrued significantly greater gains in Passage Comprehension than the control group.

An examination of individual data plotted in the upper right panel of Figure 4 demonstrates that gains achieved by the control group can be largely attributed to

\footnotetext{
${ }^{7}$ This estimate is likely to be rather conservative, given that most children did not complete or test out of all 77 lessons (See Results section, Progression Through the Intervention Materials).
} 
the improvements of two participants, whereas gains for the intervention group were more broadly distributed across the group. The pattern of individual pretest and gain scores does not appear to suggest an obvious relation between a child's pretest score and the amount of progress made over the 4 months of the intervention. Application of the learning criterion revealed that 9 of 11 children in the intervention group surpassed the learning criterion of one third grade level of improvement during the intervention as opposed to only 2 of 12 children in the control group surpassed this criterion. A chi-square comparison between the control and intervention groups demonstrated a significant effect of group assignment on the number of children passing the criterion, $\chi^{2}(4, N=23)=6.17, p<.05$.

Given the nature of the Passage Comprehension subtest, which requires children to read text and fill in missing words, at least a portion of the gains observed may reflect more accurate decoding of the words in each passage. In addition, a portion of the comprehension gains may have resulted from the tutor-directed sentence reading activities in which tutors repeatedly asked children to elaborate on the meaning of each sentence.

\section{Phonemic Awareness Measures}

Blending words. As shown in the first section of Table 4, both the intervention and the control groups demonstrated improvements on the Blending Words

TABLE 4

Pretest and Posttest Means and Standard Deviations for Raw and Standard Scores of the Word Blending, Nonword Blending, and Elision Subscales of Comprehensive Test of Phonological Processing

\begin{tabular}{|c|c|c|c|c|c|c|c|c|c|c|}
\hline \multirow[b]{4}{*}{ Group } & \multicolumn{10}{|c|}{ Measure } \\
\hline & \multicolumn{5}{|c|}{ Raw Scores } & \multicolumn{5}{|c|}{ Standard Score } \\
\hline & \multicolumn{2}{|c|}{ Pretest } & \multicolumn{2}{|c|}{ Posttest } & \multirow[b]{2}{*}{ Gain } & \multicolumn{2}{|c|}{ Pretest } & \multicolumn{2}{|c|}{ Posttest } & \multirow[b]{2}{*}{ Gain } \\
\hline & $M$ & $S D$ & $M$ & $S D$ & & $M$ & $S D$ & $M$ & $S D$ & \\
\hline \multicolumn{11}{|l|}{ Word Blending } \\
\hline Control $^{\mathrm{a}}$ & 11.5 & 3.8 & 14.3 & 4.6 & 2.8 & -0.97 & 0.62 & -0.44 & 0.77 & 0.53 \\
\hline Intervention $^{\mathrm{b}}$ & 12.5 & 2.8 & 16.6 & 4.9 & 4.1 & -0.75 & 0.55 & -0.01 & 0.94 & 0.75 \\
\hline \multicolumn{11}{|c|}{ Nonword Blending } \\
\hline Control $^{\mathrm{a}}$ & 10.3 & 2.6 & 10.0 & 4.2 & -0.3 & -0.42 & 0.52 & -0.45 & 0.83 & -0.03 \\
\hline Intervention ${ }^{\mathrm{b}}$ & 9.2 & 4.4 & 12.5 & 3.2 & 3.3 & -0.67 & 0.98 & 0.08 & 0.71 & 0.75 \\
\hline \multicolumn{11}{|l|}{ Elision } \\
\hline Control $^{\mathrm{a}}$ & 12.5 & 5.2 & 13.4 & 4.3 & 0.9 & -1.07 & 0.79 & -0.93 & 0.85 & 0.13 \\
\hline Intervention $^{\mathrm{b}}$ & 11.3 & 4.0 & 14.6 & 4.1 & 3.3 & -1.43 & 0.71 & -0.69 & 0.66 & 0.73 \\
\hline
\end{tabular}

${ }^{\mathrm{a}} n=10 .{ }^{\mathrm{b}} n=12$. 
subtest. ANOVA results for standard scores revealed a significant main effect of time, $F(1,20)=13.2, p<.001$. Although the intervention group gains were nominally larger than the control group gains, the Group $\times$ Time Span interaction term did not approach significance; thus, these data provide no evidence to suggest that the gains observed in Blending Words are linked to participation in the intervention.

Blending nonwords. Results of the Blending Nonwords test are tabulated in the second section of Table 4 . ANOVA results for standard scores revealed that improvements were significantly larger for the intervention group versus the control group, as evidenced by a significant Group $\times$ Time Span interaction, $F(1,20)=$ $4.417, p<.05$. Individual plots, presented in the lower left panel of Figure 4, demonstrate that improvements are broadly distributed across the individuals in the intervention group. These data also provide some preliminary basis for suggesting that children with above-average Nonword Blending skills might benefit less from the intervention than children with below-average skills.

Elision. As summarized in the third section of Table 4, participation in the intervention group led to larger gains in Elision test performance. This observation was substantiated by standard score ANOVA results that demonstrated a significant Group $\times$ Time interaction, $F(1,20)=5.0, p<.037$.

As illustrated in the lower right panel of Figure 4, the individual data demonstrate gains in Elision standard scores for nearly all participants in the intervention group. The two exceptions to this pattern may suggest that the intervention might be less effective at producing gains in Elision performance for children with the highest pretest scores.

Results of the three phonemic awareness measures together generally indicated some form of improvements for both groups over the time span of the intervention, yet significantly greater benefits for the intervention group on the more demanding phonemic awareness tasks. Furthermore, although the control group demonstrated some improvement over the time span of the study, such gains were apparently restricted to blending familiar words. When blending was examined in a context that increased phonological demands by using novel pseudowords, the control group's gains were significantly inferior to the gains of the intervention group. Furthermore, the control group's failure to demonstrate significant improvements on the Nonword Blending or Elision tasks suggests that the deficits demonstrated in the pretest measures are rather persistent and that whatever factors accounted for their improved skill at blending words did not lead to improvements in more demanding phonemic awareness tasks. Taken as a whole, results suggest that the intervention led to improvements in phonemic awareness skills that require the greatest degree of phonemic analysis. This pattern of results is in accord with a causally reciprocal model of the relation between decoding proficiency and phonological awareness (e.g., Perfetti et al., 1987). 


\section{DISCUSSION}

This study represents the first effort to assess the effectiveness of a particular decoding skills instructional program. Gains were observed in decoding, phonemic awareness, and passage comprehension. Comparisons with a randomly assigned waiting-list control group demonstrated that the benefits were the result of participation in the intervention. The effectiveness of this program and the magnitude of the gains might be considered very encouraging when framed in the context of an intervention study that only involved $20 \mathrm{hr}$ of instruction over 4 months delivered by minimally trained undergraduates.

Beyond providing the initial evidence for the effectiveness of Word Building, our study aimed to contribute to understanding the nature of reading difficulties and successful remediation programs. What goes wrong for children who fail to engage in full alphabetic decoding and how does this inhibit overall reading achievement? Why might an intervention that focuses a child's attention on decoding grapheme-phoneme units in all positions within words have a substantial impact on reading-related skills beyond decoding ability?

In addressing these questions, we draw on a theoretical framework of normal reading development that is shared by several theorists (i.e., Ehri, 1995, 1999; Perfetti, 1985; Share \& Stanovich, 1995). This framework views alphabetic decoding as a central skill in reading development, primarily because of the critical role that decoding plays in the process of self-teaching. The self-teaching framework holds that full alphabetic decoding (i.e., grapheme-phoneme decoding applied to each of the constituent grapheme-phoneme elements in a word) allows children to produce a close approximation of the proper pronunciation for unfamiliar words and helps them form more accurate and refined representations of words they have encountered before. Furthermore, as children gain continued experience with alphabetic decoding skill via reading experience, decoding skill itself becomes strengthened. Thus willful engagement in alphabetic decoding may serve as a boot strapping mechanism (Share, 1995) that helps readers progress from early attempts at novel words toward accurate identification of familiar words in a way that captures all the important orthographic content necessary to specify a word fully.

This framework of normal reading development is potentially useful for understanding the reading impairments observed in this study. Fine-grained analyses of grapheme and phoneme errors within word forms, such as those applied to spelling by Treiman and her colleagues (e.g., Bernstein \& Treiman, 2001), provide some potentially important observations that might help explain why the children in this study did not develop reading skill at a normal rate. The pretest results suggest that when these children encounter unfamiliar word forms, they might engage in strategies that Ehri classifies as reflecting a partially alphabetic decoding phase. Although the children might successfully decode the first letter of a novel word, they 
do not successfully apply decoding skills to each letter in a word. This pattern of results may reflect a minimal (rather than exhaustive) decoding strategy during reading, and such minimal decoding information might represent one constraint, among many, that these children use to identify words. In this way, these children might fail to benefit from the self-reinforcing aspect that typically comes from continually applying alphabetic decoding skills across all letters in a word. This account is consistent with the pattern of results demonstrated by the control group over the 4 months of observation. The effects of letter position on decoding accuracy remained highly stable during the observation period, as did overall decoding ability.

The self-teaching framework may also be useful for providing an account of the pattern of intervention results produced by the Word Building intervention. However, we should point out that such accounts are speculative in nature, as all intervention effects reported in the previous discussion are defined by contrasts with a control group that received no uniform intervention.

One implication of the framework is that intervention procedures that encourage and scaffold the process of applying partial alphabetic skills to each position within a word form should help children benefit from their reading experiences in ways that both help them to improve their general decoding skills and help them to engage in more efficient self-teaching of particular words. Based on our descriptive analysis of the progressive minimal contrast activity, we suggest that this activity might have qualities that are particularly well suited to enable and encourage children with weak decoding skills to engage in full alphabetic decoding. By continually providing children with an opportunity to form and decode a word by manipulating a single grapheme within the previously decoded word, this intervention might scaffold the process of successfully attending to and decoding each grapheme position within a word, especially those positions the child might habitually neglect, such as the medial and final positions.

Next we consider the impact of Word Building on phonological awareness measures. A key element of the framework discussed previously is the notion that decoding skills may have an important influence on other reading skills, such as phonemic awareness and comprehension. In the case of phonemic awareness, such a proposal is consistent with evidence supporting a reciprocal relation between decoding and phonological awareness (Morais, Cary, Alegria, \& Bertelson, 1979; Perfetti et al., 1987). Furthermore, the interdependent nature of the development of decoding and phonological skills provides a potentially useful explanation of how benefits in decoding ability might lead to gains in phonological awareness skills. Once reading experience begins, the process of actively decoding an alphabetic orthography may have a profound effect on the development of phonemic awareness. One potential mechanism for this influence is the increasing pressure that decoding places on the phonological system to represent phoneme units within words. The degree to which children engage (or fail to engage) in full alphabetic 
decoding may be especially important in developing phonemic awareness. Under these assumptions, to the extent that the intervention increases full alphabetic decoding, it might have a related impact on the development of phonemic awareness skills. It is equally possible that the progressive minimal contrast activities have a direct, but implicit, impact on phonemic awareness. By continually encouraging children to pronounce words that differ by a single phoneme in one of several potential positions within the word, this activity may draw the child's attention to the task of analyzing spoken words as a collection of segmentable phoneme units.

Our finding that phonemic awareness skills improve via an intervention that diverts no time away from reading instruction to engage children in special speech analysis activities may hold practical implications. Although previous studies have demonstrated support for the use of phonemic awareness as a speech-related activity practiced in isolation (Bentin \& Leshem, 1993; Hurford et al., 1994), a recently emerging consensus demonstrates that, beyond preschool, phonemic awareness instruction is most effective when it is imparted in the context of printed letters (for two recent meta-analyses, see Bus \& Ijzendoorn, 1999; National Reading Panel, 2000). It is possible that Word Building successfully integrates decoding and phonological awareness remediation within a program that focuses all the instruction time on reading skills. However, direct evaluation of such a possibility will require direct comparisons between word building and more traditional combinations of phonemic awareness and print activities.

It is also of considerable practical importance to understand the basis for the intervention-based improvements in reading comprehension. Despite strong theoretical and empirical links between decoding and comprehension (e.g., Perfetti, 1985; Shankweiler et al., 1999), producing gains in decoding abilities does not necessarily lead to gains in reading comprehension (e.g., Torgesen et al., 1999; however, see Torgesen et al., 2001, for a counterexample). The design of our study, however, provides no direct evidence that might indicate what part of the intervention is most crucial to producing the observed gains in comprehension skills. One potentially important consideration, discussed previously, is that gains in comprehension scores are directly linked to gains in decoding skill. Another consideration is that a significant portion of intervention time focused on decoding words within sentences, followed by comprehension probes. We suspect that a key ingredient for transfer to comprehension is the opportunity to practice the acquired decoding skills in texts. Such practice can solidify decoding gains and, if most of the words in a text are easily decodable, allow resources to go to other aspects of comprehension. It is possible that the integrated content across decoding and sentence activities helped encourage children to transfer their developing decoding skills to text. Clearly, whether such conjecture is correct, an important challenge for research is to identify the critical components of decoding instruction that can allow a simultaneous lifting of comprehension skills. 
To summarize, the self-teaching framework provides a useful explanatory framework for considering the pattern of pretest and intervention effects in this study. In this framework, the act of engaging in full alphabetic decoding during reading plays a central role in the development of effective and efficient word recognition skills. Efficient word decoding skills enhance reading comprehension and develop some forms of phonological awareness. A failure to engage in full alphabetic decoding would predict slower growth across each of the reading skills described. This account fits well with the pattern of deficits displayed by the children in this study, as well as the magnitude of gains obtained from the intervention. The largest gains appeared in measures that directly assessed decoding ability, and, within this skill, the greatest gains occurred for grapheme units within medial and final positions of word forms. The second largest gains appeared in the Elision task, which is closely related to alphabetic decoding (Perfetti, 1985). The third largest gains appeared in the passage comprehension measures, which are perhaps indirectly influenced by decoding ability via improvements in the efficiency of recognizing words in a text.

\section{FUTURE DIRECTIONS}

Although the previous discussion regarding the potential sources underlying the successful impact of Word Building are decidedly speculative, our study lays the groundwork for testing such proposals in future research. One such effort, currently underway, will compare Word Building activities to other decoding activities to test directly the hypothesis that progressive minimal contrast transformations provide advantages over other commonly practiced decoding activities. Depending on the outcome of such a study, the same intervention protocol can be used to construct more fine-grained decompositions of the Word Building protocol in which the presence or absence of a proposed critical feature is contrasted across two groups of participants. Such research holds the potential of going beyond gross comparisons of entire existing curricula to begin to identify specific activities that might be particularly powerful in driving improvements in targeted reading skills.

In the meantime, we have demonstrated that the Word Building intervention was useful in improving reading skills deficits for students beyond the first grade who demonstrated poor decoding skills. The instructional activities in Word Building, however, were built on insights from the normal development of early reading and have been used by Isabel Beck and her colleagues in first-grade classrooms to teach beginning reading, resulting in positive appraisals by first-grade teachers. We propose that applying these techniques early in reading instruction may encourage full alphabetic decoding from the start and potentially prevent some of the difficulties demonstrated by the children in this study. 


\section{ACKNOWLEDGMENTS}

We are grateful to the J. S. McDonnell Foundation's Cognitive Studies in Education grant (No. 98-3) that supported the work on which this article is based. The opinions expressed do not necessarily reflect the position or policy of the McDonnell Foundation, and no official endorsement should be inferred.

We express our gratitude to Linda Messineo, Maggie Gibb, and the 12 undergraduate tutors at the Learning Research and Development Center Reading Institute who provided assistance in the reading intervention. We also thank three anonymous reviewers for their insightful questions and constructive suggestions.

\section{REFERENCES}

Achenbach, T. M., \& Edelbrock, C. (1983). Manualfor the Child Behavior Checklist and Revised Child Behavior Profile. Burlington: University of Vermont, Department of Psychiatry.

Adams, M. J. (1990). Beginning to read: Thinking and learning about print. Cambridge, MA: MIT Press.

Beck, I. (1989). Reading today and tomorrow: Teachers' editions for grades 1 and 2. Austin, TX: Holt.

Beck, I., \& Hamilton, R. (2000). Beginning reading module. Washington DC: American Federation of Teachers. (Original work published 1996)

Bentin, S., \& Leshem, H. (1993). On the interaction between phonological awareness and reading acquisition: It's a two-way street. Annals of Dyslexia, 43, 125-148.

Bernstein, S. E., \& Treiman, R. (2001). Learning a novel grapheme: Effects of positional and phonemic context on children's spelling. Journal of Experimental Child Psychology, 79, 56-77.

Bus, A. G., \& Ijzendoorn, M. H. (1999). Phonological awareness and early reading: A meta-analysis of experimental training studies. Journal of Experimental Psychology, 91, 403-414.

Chall, J. S. (1987). Learning to read: The great debate. New York: McGraw-Hill. (Original work published 1967)

Conner, K. C., Sitarenios, G., Parker, J. D. A., \& Epstein, J. N. (1998). Revision and restandardization of the Conners Teacher Rating Scale (CTRS-R): Factor structure, reliability, and criterion validity. Journal of Abnormal Child Psychology, 26, 279-291.

Dunn, L. M., \& Dunn, L. M. (1981). Peabody Picture Vocabulary Test-Revised. Circle Pines, MN: American Guidance Service.

Ehri, L. C. (1991). Learning to read and spell words. In L. Rieben \& C. A. Perfetti (Eds.), Learning to read: Basic research and its implications (pp. 57-73). Hillsdale, NJ: Lawrence Erlbaum Associates, Inc.

Ehri, L. C. (1999). Phases of development in learning to read words. In J. Oakhill \& R. Beard (Eds.), Reading development and the teaching of reading: A psychological perspective (pp. 79-108). Oxford: Blackwell.

Foorman, B. A., Fletcher, J. M., Francis, D. J., Schatschneider, C., \& Mehta, P. (1998). The role of instruction in learning to read: Preventing reading failure in at-risk children. Journal of Educational Psychology, 90, 37-56.

Fowler, C. A., Liberman, I. Y., \& Shankweiler, D., (1977). On interpreting the error pattern in beginning reading. Language and Speech, 20, 162-173. 
Gough, P. B. (1991). The complexity of reading. In R. Hoffman \& D. Palermo (Eds.), Cognition and the symbolic processes: Applied and ecological perspectives (pp. 141-149). Hillsdale, NJ: Lawrence Erlbaum Associates, Inc.

Gough, P. B., \& Hoover, W. A. (1990). The simple view of reading. Reading and Writing, 2127-160.

Hurford, D. P., Schauf, J. D., Bunce L., Blaich, T., \& Moore, K. (1994). Early identification of children at risk for reading disabilities. Journal of Learning Disabilities, 27, 371-382.

Lovett, M. W., Ransby, M. J., Hardwick, N., \& Johns, M. S. (1989). Can dyslexia be treated? Treatment-specific and generalized treatment effects in dyslexic children's response to remediation. Brain and Language 37, 90-121.

Morais, J., Cary, L., Alegria, J., \& Bertelson, P. (1979). Does awareness of speech as a sequence of phones arise spontaneously? Cognition, 7, 323-331.

National Reading Panel. (2000). Teaching children to read: An evidence-based assessment of the scientific research literature on reading and its implications for reading instruction. Washington, DC: National Institute of Child Health and Human Development.

Perfetti, C. A. (1985). Reading ability. New York: Oxford University Press.

Perfetti, C. A. (1991). Representations and awareness in the acquisition of reading competence. In L. Rieben \& C. A. Perfetti (Eds.), Learning to read: Basic research and its implications (pp. 33-44). Hillsdale, NJ: Lawrence Erlbaum Associates, Inc.

Perfetti, C. A., Beck, I., Bell, L., \& Hughes, C. (1987). Phonemic knowledge and learning to read are reciprocal: A longitudinal study of first grade children. Merrill-Palmer Quarterly, 33, 283-319.

Shankweiler, D., Lundquist, E., Katz, L., Stuebing, K. K., Fletcher, J. M., Brady, S., et al. (1999). Comprehension and decoding: Patterns of association in children with reading difficulties. Scientific Studies of Reading, 3, 69-94.

Share, D. L. (1995). Phonological recoding and self-teaching: Sine qua non of reading acquisition. Cognition, 55, 151-218.

Share, D. L., \& Stanovich, K. E. (1995). Cognitive processes in early reading development: Accommodating individual differences into a model of acquisition. Issues in Education, 1, 1-57.

Snow, C. E., Burns, M. S., \& Griffin, P. (1998). Preventing reading difficulties in young children. Washington, DC: National Academy Press.

Stanovich, K. E. (1991). Word recognition: Changing perspectives. In R. Barr \& M. Kamil (Eds.), Handbook of reading research. (Vol. 2, pp. 418-452). Hillsdale, NJ: Lawrence Erlbaum Associates, Inc.

Torgesen, J. K. (in press). Lessons learned from intervention research in reading: A way to go before we rest. In R. Stainthrop \& P. Tomlinson (Eds.), Learning and teaching reading. Leicester, England: British Psychological Society.

Torgesen, J. K., Alexander, A. W., Wagner, R. K., Rashotte, C. A., Voeller, K., Conway, T., et al. (2001). Intensive remedial instruction for children with severe reading disabilities: Immediate and long term outcomes from two instructional approaches. Journal of Learning Disabilities, 34, 33-58.

Torgesen, J. K., Wagner, R. K., Rashotte, C. A., Rose, E., Lindamood, P., Conway, T., et al. (1999). Preventing reading failure in failure in young children with phonological processing disabilities: Group and individual responses to instruction. Journal of Educational Psychology, 91, 579-593.

Wagner, R. K., Torgesen, J. K., \& Rashotte, C. A. (1999). The Comprehensive Test of Phonological Processing. Austin, TX: PRO-ED.

Woodcock, R. N. (1998). Woodcock Reading Mastery Tests-Revised. Circle Pines, MN: American Guidance Service.

Manuscript received April 2, 2001

Accepted October 25, 2001 